\title{
TOXIDEZ POR ALUMÍNIO E HIDROGÊNIO NO CRESCIMENTO DE RAÍZES DE MILHO
}

\author{
VERA MARIA CARVALHO ALVES ${ }^{1}$, GILSON VILLAÇA EXEL PITTA ${ }^{1}$, SIDNEY NETO \\ PARENTONI ${ }^{1}$, ROBERT EUGENE SCHAFFERT ${ }^{1}$, ANTÔNIO MARCOS COELHO ${ }^{1}$, JURANDIR \\ VIEIRA DE MAGALHÃES ${ }^{1}$
} ${ }^{1}$ Pesquisadores, Embrapa Milho e Sorgo. Caixa Postal 151, CEP. 35701-970 Sete Lagoas, MG. E-mail:
vera@cnpms.embrapa.br (autora para correspondência).

Revista Brasileira de Milho e Sorgo, v.3, n.2, p.311-318, 2004

\begin{abstract}
RESUMO - Com o objetivo de se investigar a tolerância diferencial de linhagens de milho ao alumínio (Al) e sua interação com alta atividade de prótons, em solução nutritiva, foram conduzidos dois experimentos em casa de vegetação. No primeiro experimento foram avaliadas seis linhagens de milho, provenientes do Programa de Melhoramento da Embrapa Milho e Sorgo na presença de quatro concentrações de Al $(0,55,111$ e $222 \mu \mathrm{M})$ e no segundo, as mesmas linhagens foram avaliadas em quatro diferentes valores de $\mathrm{pH}$ da solução nutritiva $(3,8,4,2,4,6$ e 5,0). No transplantio e durante os cinco dias de tratamento, foram medidos os comprimentos das raízes seminais das plântulas em todos os tratamentos. Das linhagens avaliadas, quatro foram tolerantes ao $\mathrm{Al}$ (Cateto Al 237, SLP 181, L 1154, L 3) e duas sensíveis (L 53 e L 36). Não foi observada uma correlação definida entre tolerância ao Al e tolerância a alta atividade de prótons em solução nutritiva. A característica de tolerância foi melhor avaliada na concentração de $222 \mu \mathrm{M}$ de Al, sugerindo-se esta concentração em trabalhos futuros de seleção de genótipos na solução nutritiva utilizada. Verificou-se também que o $\mathrm{pH}$ 4,2 foi o mais adequado para as condições estudadas.
\end{abstract}

Palavras-chave: tolerância a alumínio, toxidez de alumínio, adaptação a solos ácidos, Zea mays.

\section{TOXICITY CAUSED BY ALUMINIUM AND HIDROGEN ON THE GROWTH OF MAIZE ROOT}

\begin{abstract}
With the objective of investigating differential tolerance of maize inbred lines to aluminum (Al) and its interaction with high proton activity, in nutritive solution, two experiments were carried out in green houses. In the first experiment, six maize inbred lines from EMBRAPA's breeding program were evaluated in four different $\mathrm{Al}$ concentrations $(0,55,111$ and $222 \mu \mathrm{M})$ and in the second experiment, the same lines were evaluated in nutritive solution with four different $\mathrm{pH}$ values $(3.8,4.2,4.6$ and 5.0). During transplant and five days of treatment, seminal root length was measured for all treatments. From the inbred lines evaluated, four were Al tolerant (Cateto Al 237, SLP 181, L 1154, L3) and two were sensitive (L 53 and L 36). There was no defined correlation between $\mathrm{Al}$ tolerance and tolerance to high proton activity in nutritive solution. The tolerance characteristic was better evaluated in the concentration of $222 \mu \mathrm{M}$ of Al, suggesting
\end{abstract}


that this concentration will be utilized in future studies of genotype selection. It was also verified that the $\mathrm{pH}$ value of 4.2 was more adequate for the present study conditions.

Key words: aluminum tolerance, aluminum toxicity, acid soils adaptation, Zea mays.

A toxidez por alumínio (Al) é considerada um importante fator que limita a produção das culturas em solos ácidos. Em valores de $\mathrm{pH}$ do solo iguais ou inferiores a 5,0, a espécie trivalente $\left(\mathrm{Al}^{3+}\right)$ considerada a mais tóxica prevalece na solução do solo, inibindo o crescimento de raízes. O efeito sobre a planta resulta em um sistema radicular pouco desenvolvido, limitando a exploração das camadas mais profundas de solo, restringindo a absorção de água e de nutrientes. A acidez do solo reduz a produtividade agrícola em diversas regiões do mundo, principalmente em solos tropicais. Aproximadamente $30 \%$ das terras agriculturáveis do mundo são ácidas (von Uexküll \& Mutert, 1995, citados por Kochian et al., 2002).

O primeiro sintoma de toxidez de Al é a inibição da elongação da raiz, que ocorre cerca de 1-2 h após a exposição a Al (Kochian, 1995). Por essa razão, a avaliação do crescimento radicular tem sido amplamente utilizada como indicadora de tolerância ao íon $\mathrm{Al}$, principalmente em experimentos de seleção de genótipos realizados em solução nutritiva. Kollmeier et al. (2000) apresentaram evidências de que os mecanismos primários de tolerância a $\mathrm{Al}$ em genótipos de milho estão localizados na zona distal de transição da raiz e sugeriram uma rota de sinalização localizada no ápice radicular, mediando o sinal de Al entre a zona distal de transição e a zona de elongação por meio de transporte basipetal de auxinas.

$\mathrm{Na}$ maioria dos solos ácidos com $\mathrm{pH}$ superior a 4, a alta concentração de $\mathrm{Al}$ é provavelmente um fator mais importante para limitar a produtividade do que a alta atividade de prótons.
Estudos têm mostrado a interação entre toxidez de $\mathrm{Al}$ e de prótons em diferentes culturas como soja, feijão (Lazof \& Holland, 1999) e milho (Llugany et al., 1995; Gunsé et al., 1997), enfatizando a necessidade de se caracterizar genótipos nestas duas situações para o melhor entendimento dos mecanismos de tolerância.

O objetivo deste trabalho foi o de investigar a tolerância diferencial de linhagens de milho ao $\mathrm{Al}$ e sua interação com alta atividade de prótons, em solução nutritiva.

A avaliação dos genótipos de milho foi realizada por meio da condução de dois experimentos:

Experimento 1. Foram avaliadas seis linhagens de milho, provenientes do programa de melhoramento da Embrapa Milho e Sorgo (Cateto Al 237, L 1154, L 3, SLP 181, L 36 e L 53) na presença de quatro concentrações de $\mathrm{Al}(0,55,111$ e 222 $\mu \mathrm{M})$. Após desinfecção com solução de hipoclorito de sódio a 5\% de concentração, por $15 \mathrm{~min}$, sementes das seis linhagens de milho foram colocadas para germinar em rolos de papel toalha, parcialmente imersos em água deionizada, por cinco dias. Após a germinação, plântulas de cada linhagem foram transferidas para bandejas plásticas contendo $8,5 \mathrm{~L}$ de solução nutritiva de Clark, adaptada por Magnavaca (1982), pH 4.0. Decorridas 24 horas, a solução nutritiva foi substituída por outra, de constituição idêntica, contendo as quatro concentrações de $\mathrm{Al}$, na forma de $\mathrm{Al}_{2} \mathrm{~K}\left(\mathrm{SO}_{4}\right)$. O comprimento da raiz seminal foi medido imediatamente antes da troca de solução nutritiva e após 1, 2, 3, 4 e 5 dias de tratamento. O experimento foi composto por um fatorial $6 \times 4$ (seis linhagens e quatro doses de 
Al), distribuído em delineamento inteiramente casualizado, com três repetições. Cada repetição foi composta por sete plantas.

Experimento 2. Foram avaliadas as mesmas linhagens de milho do experimento anterior, na presença de quatro valores de $\mathrm{pH}(3,8,4,2,4,6 \mathrm{e}$ $5,0)$. A desinfeção, germinação das sementes, solução nutritiva utilizada, delineamento experimental e repetições foram similares ao experimento anterior. $\mathrm{O}$ comprimento da raiz seminal foi medido imediatamente antes do transplantio e após 5 dias de tratamento.

Verificou-se que as linhagens Cateto $\mathrm{Al}$ 237, L 1154, L 3 e SLP 181 não apresentaram redução de crescimento da raiz seminal, nas doses de 55 e $111 \mathrm{mM}$ de $\mathrm{Al}$, quando comparadas com a dose zero (Figura 1). Na maior dose, 222 $\mu \mathrm{M}$ de Al, as linhagens Cateto Al 237 e SLP 181 também tiveram o crescimento da raiz seminal inalterado em relação à dose zero, sendo que as linhagens L 1154 e L 3, apresentaram pequena redução ( $18,7 \%$ e $4,3 \%$, respectivamente). Observou-se, também, que somente a linhagem L 53 se mostrou sensível ao $\mathrm{Al}$ em todas as doses estudadas, sendo que a linhagem L 36 mostrouse sensível somente nas doses mais altas de $\mathrm{Al}$ (111 e $222 \mu \mathrm{M})$.

Pelo comportamento dos materiais, observou-se que a dose de $222 \mu \mathrm{M}$ de Al permitiu a melhor distinção entre os materiais tolerantes e sensíveis, visto que, nas doses mais baixas, verificou-se um estímulo do crescimento radicular, principalmente das linhagens SLP 181 e L 3 (Figura 1). As linhagens Cateto Al 237 e SLP 181 foram as mais tolerantes ao $\mathrm{Al}$, sem redução no crescimento radicular, seguidas pela L 3 e L 1154, com pequenas reduções (4 e $18 \%$, respectivamente). As linhagens L 36 e L 53 foram as mais sensíveis ao $\mathrm{Al}$, com redução no crescimento do sistema radicular de 65 e $74 \%$, respectivamente, em relação à testemunha (Figura 1).
Aumentos transitórios na taxa de crescimento radicular em doses sub-tóxicas de $\mathrm{Al}$ têm sido observados em diferentes espécies de plantas como arroz, milho e algumas dicotiledôneas, sendo geralmente mais evidentes nas cultivares tolerantes que nas sensíveis. Estímulo no crescimento das raízes de várias espécies quando na presença de baixas concentrações de Al tem sido atribuído a alterações na distribuição de substâncias de crescimento e prevenção da toxidez de cobre e manganês (Llugany et al., 1995). Trabalhos conduzidos por Kinraide et al. $(1992,1994)$ demonstram que o cálcio pode desempenhar um papel específico no alívio da toxidez devido à alta atividade de prótons, mas que esse efeito pode também ser desempenhado por outros cátions, inclusive pelo $\mathrm{Al}$. $\mathrm{O}$ íon $\mathrm{Al}^{3+}$, por ser um cátion trivalente, pode reduzir a negatividade da superfície celular e, conseqüentemente, reduzir a atividade do $\mathrm{H}^{+}$na superfície da membrana celular. Entretanto, esse estímulo sobre o crescimento é um fenômeno dependente de tempo, podendo a toxidez se manifestar após determinado período de tratamento, fato observado na Figura 2. No quinto dia de tratamento com $\mathrm{Al}$, o efeito do estímulo de crescimento nas doses de 55 e $111 \mu \mathrm{M}$ de $\mathrm{Al}$ foi completa ou parcialmente revertido nas linhagens L 3 e SLP 181.

As linhagens de milho mais sensíveis ao baixo $\mathrm{pH}$ foram L 36, L 3, L 53 e L 1154, que aumentaram o crescimento radicular em 30, 27, 22 e $19 \%$, respectivamente após cinco dias de tratamento, quando o $\mathrm{pH}$ da solução nutritiva aumentou de 3,8 para 4,2 (Figura 3). A linhagem L 3 aumentou o crescimento da raiz seminal em $40 \%$ quanto o $\mathrm{pH}$ passou de 3,8 para 4,6 . O crescimento da raiz seminal da linhagem SLP 181 não foi afetado pelo $\mathrm{pH}$ da solução, enquanto o da Cateto aumentou $18 \%$ quando o $\mathrm{pH}$ passou de 4,6 para 5,0. 

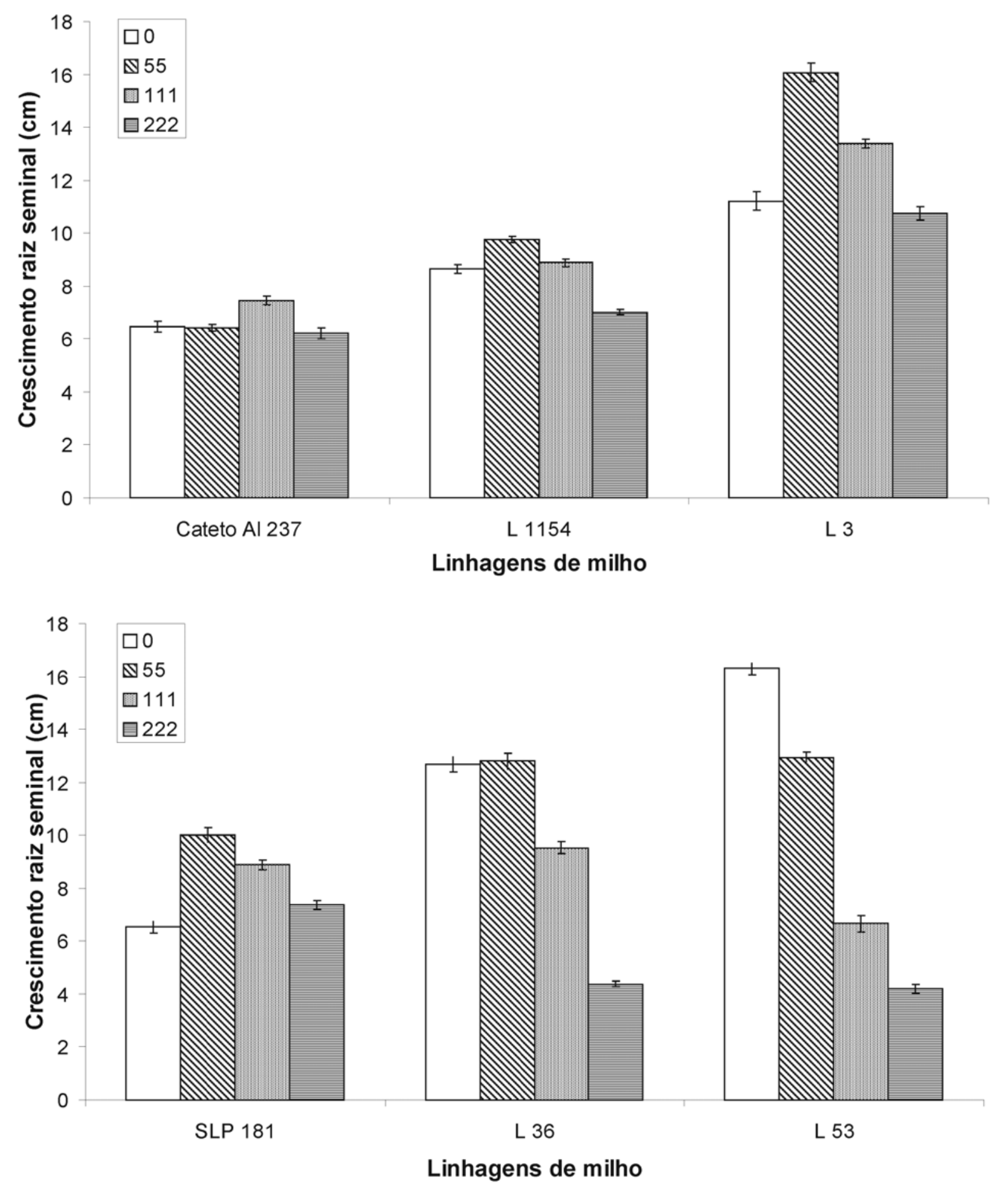

FIGURA 1. Crescimento de raiz seminal $(\mathrm{cm})$ em solução nutritiva, $\mathrm{pH} 4,0$, de seis linhagens de milho (Cateto, L1154, L3, SLP 181, L36 e L53), após cinco dias de tratamento com 0, 55, 111 e $222 \mu \mathrm{M}$ de Al. 

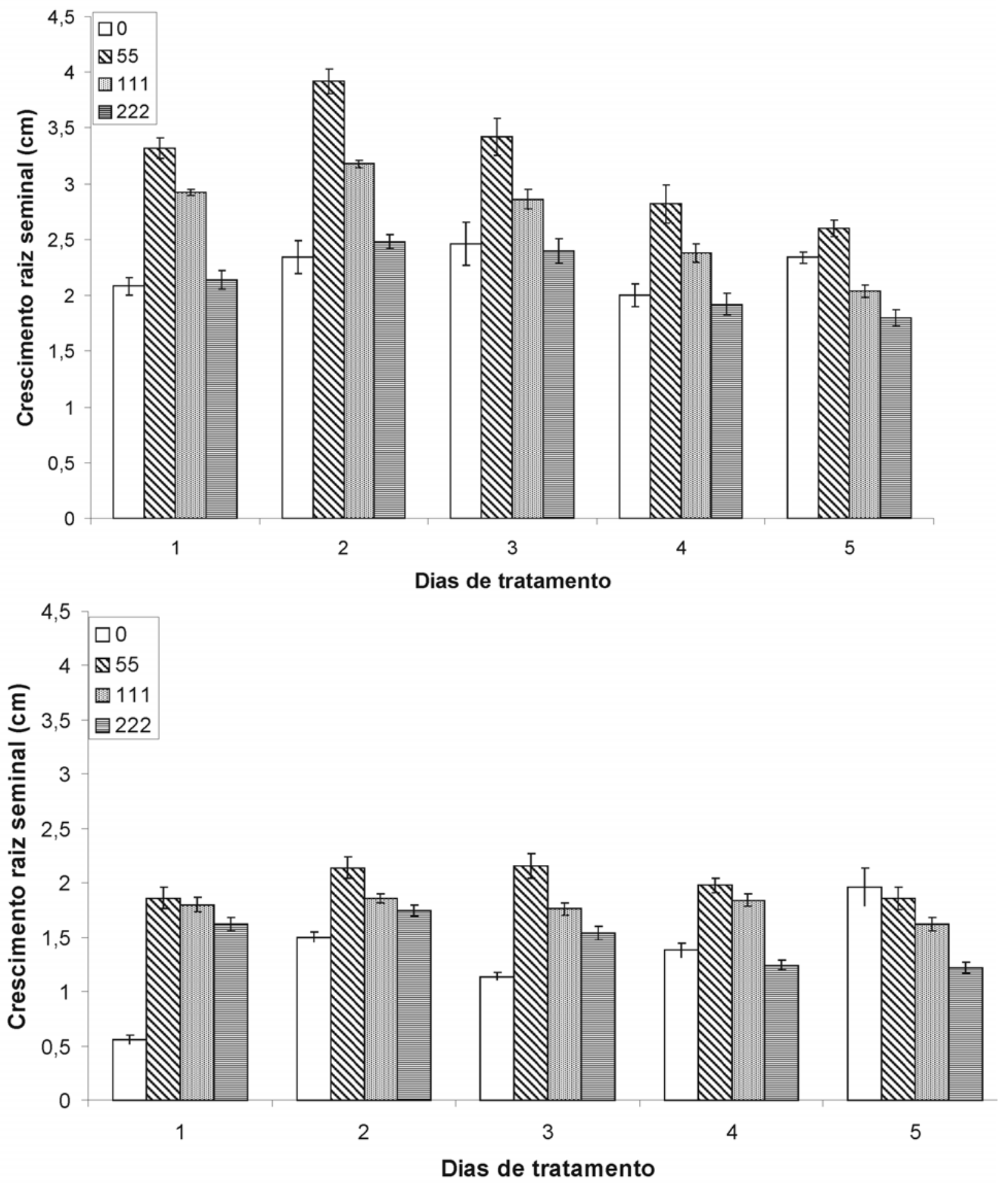

FIGURA 2. Crescimento diário da raiz seminal (cm) das linhagens de milho L 3 (A) e SLP 181 (B), em solução nutritiva, $\mathrm{pH} 4,0$, nas concentrações de $0,55,111$ e $222 \mu \mathrm{M}$ de Al. 

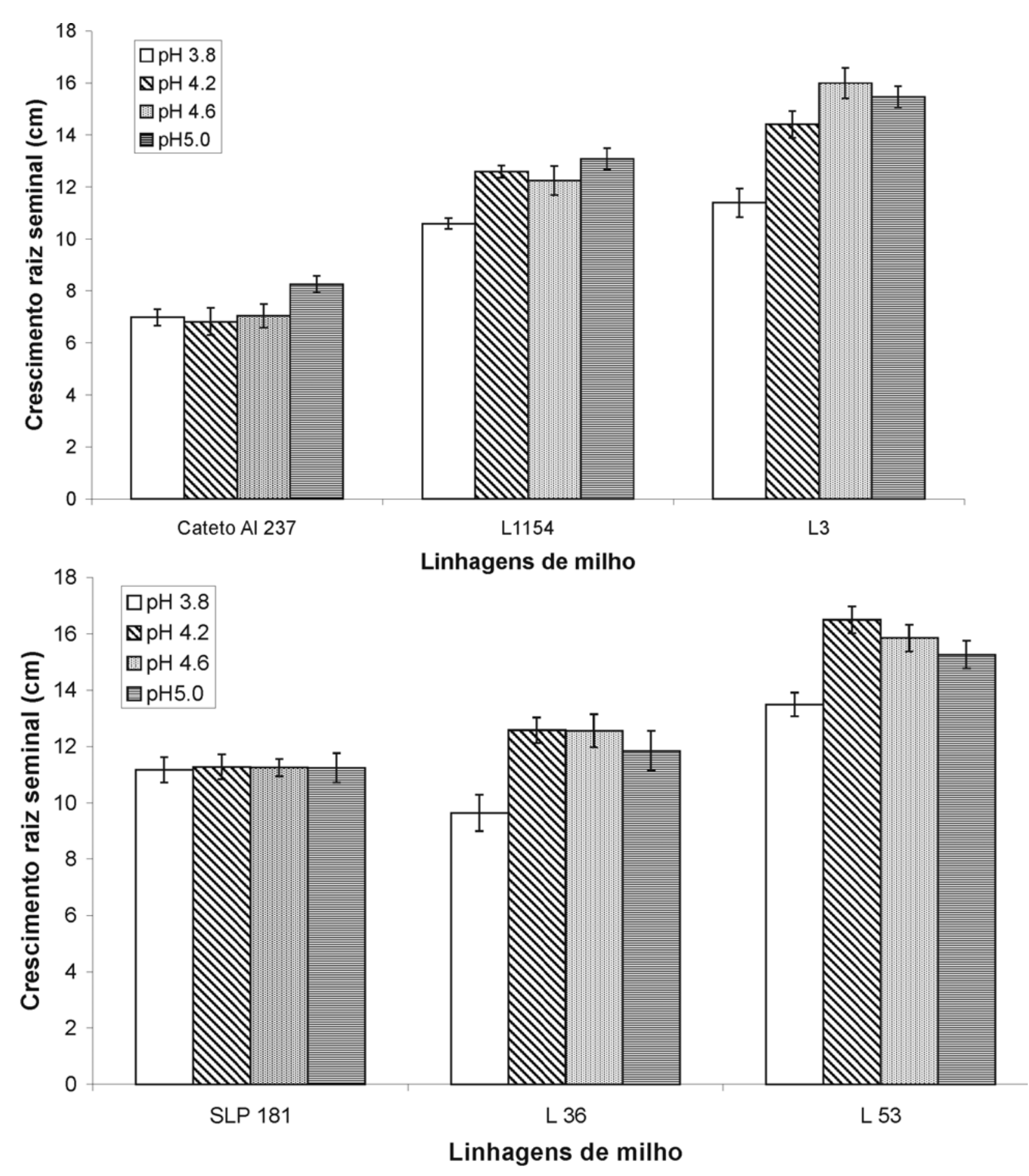

FIGURA 3. Crescimento da raiz seminal $(\mathrm{cm})$ em solução nutritiva de linhagens de milho tolerantes (Cateto, L1154, L3, SLP 181) e sensíveis (L36 e L53) ao Al, em diferentes valores de pH (3,8, 4,2, 4,6 e 5,0), após cinco dias de tratamento. 
Pelos resultados apresentados, não foi observada correlação definida entre tolerância ao Al e tolerância à alta atividade de prótons em solução nutritiva, visto serem as linhagens L 3 e L 1154 tolerantes a Al e as linhagens L 36 e L 53, sensíveis.

Com relação à hipótese de que o íon $\mathrm{Al}^{3+}$, por ser um cátion trivalente, pode reduzir a negatividade da superfície celular e, conseqüentemente, reduzir a atividade do íon $\mathrm{H}^{+}$nesta região, provocando estímulo temporário no crescimento radicular, observou-se que a linhagem L 3 apresentou essa característica devido ao estímulo de crescimento observado nas doses de 55 e $111 \mu \mathrm{M}$ de $\mathrm{Al}$ e por possuir sensibilidade a alta atividade de prótons. Por outro lado, a linhagem SLP 181, que apresentou estímulo ao crescimento radicular, foi praticamente insensível a diferentes valores de $\mathrm{pH}$ da solução nutritiva.

Os resultados obtidos permitiram as seguintes conclusões:

a) A característica de tolerância foi melhor avaliada na concentração de $222 \mu \mathrm{M}$ de Al, sugerindo esta dose em trabalhos futuros de seleção de genótipos em solução nutritiva;

b) Não foi observada correlação definida entre tolerância ao íon $\mathrm{Al}^{3+}$ e o íon $\mathrm{H}^{+}$, haja vista que algumas linhagens caracterizadas como tolerantes ao $\mathrm{Al}$ não modificaram esta característica com a variação do $\mathrm{pH}$ da solução;

c) $\mathrm{O}$ pH 4,2 se mostrou adequado para seleção de genótipos de milho quanto à tolerância ao Al, em solução nutritiva.

\section{Literatura Citada}

GUNSÉ, B.; POSCHENRIEDER, C.; BARCELÓ, J. Water transport properties of roots and root cortical cells in proton- and Al-stressed maize varieties. Plant Physiology, Bethesda, v.113, p. 595 602, 1997.
LAZOF D. B.; HOLLAND, M. J. Evaluation of the aluminum-induced root growth inhibition in isolation from low $\mathrm{pH}$ effects in Glycine max, Pisum sativum and Phaseolus vulgaris. Australian Journal of Plant Physiology, Victoria, v. 26, p. 147-157, 1999.

KINRAIDE, T. B.; RYAN, P. R.; KOCHIAN. L. V. Interactive effects of $\mathrm{Al}^{3+}, \mathrm{H}^{+}$, and other cations on root elongation considered in terms of cellsurface electrical potential. Plant Physiology, Bethesda, v. 99, p. 1461-1468, 1992.

KINRAIDE, T. B.; RYAN, P. R.; KOCHIAN. L.V. $\mathrm{Al}^{3+}-\mathrm{Ca}^{2+}$ interactions in aluminum rhizotoxicity. II. Evaluating the $\mathrm{Ca}^{2+}$-displacement hypothesis. Planta, Berlin, v. 192, p. 104-109, 1994.

KOCHIAN. L. V. Cellular mechanisms of aluminium toxicity and resistance in plants. Annual Review of Plant Physiology and Plant Molecular Biology, Palo Alto, v. 46, p. 237-260, 1995.

KOCHIAN, L. V.; PENCE, N. S.; LETHAM, L. D.; PINEROS, M. A.; MAGALHÃES, J. V.; HOEKENGA, O. A.; GARVIN, D .F. Mechanisms of metal resistance in plants: aluminum and heavy metals. Plant Soil, The Hague, v. 247, p.109-119, 2002.

KOLLMEIER, M.; FELLE, H. H.; HORST, W. Genotypical differences in aluminum resistance of maize are expressed in the distal part of the transition zone. Is reduced basipetal auxin flow involved in inhibition of root elongation by aluminum? Plant Physiology, Bethesda, v. 122, p. $945-956,2000$.

LLUGANY, M.; POSCHENRIEDER, C.; BARCELÓ, J. Monitoring of aluminium inhibition of root elongation in four maize cultivars differing in tolerance to aluminium and 
proton toxicity. Physiologia Plantarum, (Zea mays L.). 1982. 135 f. Thesis (Doctor of Copenhagen, v. 93, p. 265-271, 1995. Phylosophy in Agronomy) - University of

MAGNAVACA, R. Genetic variability and the Nebraska, Lincoln.

inheritance of aluminum tolerance in maize 